\title{
Methodologies for Improved Tag Cloud Generation with Clustering
}

\author{
Martin Leginus, Peter Dolog, Ricardo Lage, and Frederico Durao \\ Department of Computer Science, Aalborg University, \\ Selma Lagerlofs Vej 300 \\ \{mleginus, dolog, riclage\}@cs.aau.dk, freddurao@gmail.com \\ http://iwis.cs.aau.dk/
}

\begin{abstract}
Tag clouds are useful means for navigation in the social web systems. Usually the systems implement the tag cloud generation based on tag popularity which is not always the best method. In this paper we propose methodologies on how to combine clustering into the tag cloud generation to improve coverage and overlap. We study several clustering algorithms to generate tag clouds. We show that by extending cloud generation based on tag popularity with clustering we slightly improve coverage. We also show that if the cloud is generated by clustering independently of the tag popularity baseline we minimize overlap and increase coverage. In the first case we therefore provide more items for a user to explore. In the second case we provide more diverse items for a user to explore. We experiment with the methodologies on two different datasets: Delicious and Bibsonomy. The methodologies perform slightly better on bibsonomy due to its specific focus. The best performing is the hierarchical clustering.
\end{abstract}

\section{Introduction}

Tags are textual labels users use in social web sites, such as Flickr, Technorati and del.icio.us, for annotating information resources. They can be assigned freely to these resources to help remind a user of the intended meaning to him or her. Tags are also typically aggregated by social web sites into tag clouds. These are visual elements that highlight certain tags by different users, assisting them in the navigation of the web site [14,19. However, the tags in the tag clouds are mostly presented alphabetically, with their font size weighted according to their frequency. These popularity based tag clouds [21,19,6] have at least the following limitations:

- First, syntactical variations of tags i.e., typos, singular and plural forms and compounded tags that share the same semantical meaning are not addressed. Therefore, the coverage of a tag cloud is lower as syntactically different tags are not aggregated.

- Moreover, a tag cloud can contain syntactical variations of the same tag which leads to redundancy. 
- Finally, the most popular tags typically have a broader meaning, covering redundantly a certain set of documents which results into a low diversity of tags.

To address these limitations and attempt to improve the quality of tag clouds, we experiment with different clustering techniques. Due to the resource demanding nature of quality assessment of tag clouds performed by users, 23. introduced synthetic measures of quality based on tag cloud properties, such as coverage and overlap. Coverage measures a proportion of documents tagged with a particular tag with respect to the all considered documents. The overlap is a measure which captures to which extent different tags considered in comparison are associated the same resources and what is the proportion of the same resources with respect to all considered resources. In our previous paper [4] we have already shown that a specific kind of clustering, spectral clustering, has an effect on tag cloud generation and on its properties such as coverage, relevance, and overlap.

The contributions of this paper can be summarized as follows:

- We propose two new methodologies on how to integrate clustering into tag cloud generation methods, further improving coverage and overlap.

- Within the second methodology, we compare four different clustering algorithms and show that the hierarchical clustering performs the best.

- We introduce a new synthetic metric - chained coverage - which combines coverage and overlap together.

We experiment on two different datasets, Delicious and Bibsonomy. The first one is a general purpose collaborative resource tagging system. The second one is a specific tagging system for scientific literature. We show that on the Delicious dataset, the hierarchical clustering algorithm obtained a $12.4 \%$ improvement on coverage over the second best performing algorithm we tested.

The remainder of this paper is organized as follows. Section 2 positions our work with respect to related findings in the literature. Section 3 provides a background on clustering techniques we are embedding in the studied methodologies. Section 4 describes our contribution in two methodologies we have designed for the generation of tag clouds. Section 5 describes experimental set up and results which we gained from the experiments. Section 6 summarizes the paper achievements and roadmap to future work.

\section{Related Work}

Research on tag clouds has concentrated on methods for rendering and presentation aspects [2,19]. In [19], the authors perform a number of experiments to assess the effects of semantic versus alphabetical and random arrangements of tags in tag clouds. The outcome shows that clustered tag clouds can provide improvements over random layouts in specific search tasks and that they tend to increase the attention toward tags in small fonts compared to other layouts. They also claim that tag cloud layout does not seem to influence the ability 
to remember tags. Our focus is different. We decouple the presentation aspects from the selection of tags for the tag clouds generation. We study and apply clustering but we experiment with methodologies on how to combine clustering into the process of selection and generation of the tag cloud and not only in its rendering process. By doing so we enable a combination of different methods for tag selection generation and tag cloud rendering.

Random and alphabetical ordering of tags in a tag cloud was tested in 6]. Hierarchical browsing [15] looks at how to structure the layout of a tag cloud. Studies on the font size, weight, intensity and position of tags in the cloud are studied in 3,11,18. In this paper we study the selection of tags for tag cloud generation. We implement a popularity and coverage based visualization method which renders the tag cloud as one approach and not as a main goal of research. However, the aforementioned approaches can be utilized for presentation of tags selected for a tag cloud in our approach as well.

Sinclair et al. 21] consider tag clouds as useful retrieval interface when a user's searching task is not specific. In order to build the tag cloud, their approach results in displaying the top 70 most-used tags in the database. The size of each tag in the cloud is proportional to the log of its frequency, which follow a power law distribution. In our work, 8 tag clouds varying in number of tags enclosed are tested. We try to find out which number of tags in the tag cloud improved the tag cloud properties the most, which is a new findings in comparison to [21. Further, although the size of each tag is not considered for rendering purposes, the tag relevancy is calculated and determined by its popularity in the corpus, i.e. the most frequent tags are more relevant over the less popular ones. We extend the popularity/usage based approaches for tag selection with clustering and as we also show in experiments with improvements.

20. the authors deal with tag redundancy and ambiguity through a hierarchical agglomerative clustering of tags for recommendations. Hierarchical clustering is studied in our work as well as is the tag redundancy in the syntactical preclustering using the Levenhstein distance. However, the focus and end product is different from the work of [20], as is the methodology where we apply the clustering.

23. proposes different tags selection algorithms for tag clouds. Our method differ in the way the selection is performed. 23] do not apply clustering. Our objective function for computing clusters and representative tags for the tag clouds in the second methodology also differ. As a result, our generated tag clouds result in a more diverse tags thus a user can explore and browse more topics from the generated tag cloud.

[74] propose to group semantically related tags and depict them in a tag cloud near by with similar color. Such approach provides better orientation in the tag cloud as related tags can be easier identified by users. Tags are clustered based on their co-occurrences. Similarly, our proposed tag cloud generation method also groups similar tags. However, our approach is more robust as syntactically similar tags are firstly pre-clustered (grouping singular, plural or misspellings of tags) similarly as proposed in 22 . It leads to tag space reduction as resulted tag 
cloud does not contain syntactically similar tags. In the second phase, tags are similarly clustered based on tags co-occurrences but our proposed approach also considers retrieved semantic distances from WordNet dictionary if available.

12. benefit from "syntagmatic" relations based on co-occurrences similarity and subsequent clustering to generate semantically enhanced tag cloud. The elementary tasks of their approach are the calculation of tag similarities and subsequent clustering. Our work differs, first methodologically as a combination of clustering and popularity, and second as a study to find out which clustering approach performs the best.

\section{Clustering Techniques}

In the following section, we present several clustering techniques that are utilized in the process of tag cloud generation. Firstly, we introduce syntactical pre-clustering based on Levenhstein distance. Then, we present three different clustering techniques. The first two proposed approaches(Correlated Feature Hashing and Complete linkage hierarchical clustering) cluster tags according to their co-occurrence based similarities. The third (K-means) algorithm considers each tag from a tag space as feature vector. These techniques were proposed and described in [16].

\subsection{Syntactical Pre-clustering}

Syntactical pre-clustering filters out items with typographical misspellings unnecessary plural and singular forms of the same item and also compounded items from two different terms connected with some separator. These redundant items would occupy an item set unnecessarily as they would have the same semantical meaning. Levenhstein distance is first computed for each term pair from the initial term space. The distance between two terms measures the number of required changes (substitution, insertion and deletion of a character are allowed operations) to transform one term into another. We justify its use because it attains significantly better results than Hamming distance as shown in [5].

\subsection{Correlated Feature Hashing}

We propose to reduce a tag space with hashing function that is similar to the proposed technique in [1] where authors successfully reduced dictionary size by utilizing hashing. The idea is to share and group tags with similar meaning.

We sort the tags used within the system according to the frequency of usage such that $t_{1}$ is the most frequent tag and $t_{T}$ is the least frequent. For each tag $t_{i} \in 1, \ldots, T$ is calculated $D I C E$ coefficient with respect to each tag $t_{j} \in 1, \ldots, K$ among the top $K$ most frequent tags. The DICE coefficient is defined as:

$$
\operatorname{DICE}\left(t_{i}, t_{j}\right)=\frac{2 \cdot \operatorname{cocr}\left(t_{i}, t_{j}\right)}{\operatorname{ocr}\left(t_{i}\right)+\operatorname{ocr}\left(t_{j}\right)}
$$


where $\operatorname{cocr}\left(t_{i}, t_{j}\right)$ denotes the number of co-occurrences for tags $t_{i}$ and $t_{j}, \operatorname{ocr}\left(t_{i}\right)$ and $\operatorname{ocr}\left(t_{j}\right)$ is the total number of tag $t_{i}$ and $t_{j}$ assignments respectively. For each tag $t_{i}$, we sort the $K$ scores in descending order such that $S_{p}\left(t_{i}\right) \in 1, \ldots, K$ represents the tag of the p-th largest DICE score DICE $\left(t_{i}, S_{p}\left(t_{i}\right)\right)$. We can then use hash kernel approximation defined as:

$$
\overline{\Phi_{t_{j}}}(x)=\sum_{t_{i} \in T: h\left(t_{i}\right)=t_{j}} \Phi_{t_{i}}(x)
$$

and given by a hash function:

$$
h\left(t_{i}\right)=S_{1}\left(t_{i}\right)
$$

The described approach is replacing each tag $t_{i}$ with the tag $S_{1}\left(t_{i}\right)$. We have reduced tag space from all $T$ tags to the $K$ most frequent tags.

\subsection{Complete Linkage Hierarchical Clustering}

In the second approach we utilize Complete linkage agglomerative hierarchical clustering technique [10. In the beginning, each entry that should be clustered is considered as single cluster. For each cluster is computed Dice similarity (see Formula 1) with all other clusters. The cluster with the highest similarity to the considered cluster is merged with the cluster. When clusters contain more tags, the lowest similarity between two tags from those clusters is considered for the merging step. The aggregation of clusters repeats until the single cluster is obtained. The final clustering structure is denoted also as dendrogram. The required number of clusters is obtained by cutting a dendrogram at a certain level such that a given number of clusters is obtained.

\subsection{K-means}

The following clustering technique differs from the previous in such a way that each tag is expressed in $n$-dimensional vector space where the $i$-th dimension corresponds to the $i$-th item res $_{i}$ (in a similar way as in [17, 9]).

We denote $T=\left\{t_{1}, t_{2} \ldots, t_{|T|}\right\}$ as the set of all distinct tags that are clustered and $R=\left\{r e s_{1}\right.$, res $\left._{2} \ldots r e s_{n}\right\}$ the set of all items that are tagged with tags from $T$. Let $f\left(t, r e s_{i}\right)$ be equal to a frequency of a tag $t$ assigned to item res $_{i}$ otherwise it is equal to 0 . Then, the vector representation of tag $t$ is:

$$
t=\left(f\left(t, r e s_{1}\right), f\left(t, r e s_{2}\right), \ldots, f\left(t, r e s_{n}\right)\right)
$$

Once, tags from $T$ are expressed as $n$-dimensional vectors, we proceed with the cluster analysis. The K-means is a simple well known clustering technique that groups objects from a given set into $k$ clusters (given a priori). The clustering of a tag space with the K-means algorithm is computed as follows: 
1. Each tag from a tag space $T$ is expressed as $n$-dimensional vector. According to the size of the tag space and user requirements an amount of clusters is set to $k$.

2. It randomly places $k$ centroids such that a distance from each other is maximized.

3. Each tag from the tag space is bound to the nearest centroid.

4. New centroids are computed as the mean value of tags vectors grouped with a given centroid. It continues with the step 3, until new centroids are identical with the centroids from the previous iteration.

We obtained $k$ disjoint clusters of tags so we can proceed with the selection of tags for the tag cloud generation. The results of K-means algorithm depend on used distance measure - we exploit only Cosine distance as it attains the best results [16.

\section{Methodologies for Improved Tag Cloud Generation with Clustering}

In this section, we present common metrics that measure different aspects of a tag cloud. Next, we introduce our two methodologies for tag cloud generation that attempts to improve the tag clouds according to the presented metrics.

\subsection{Tag Cloud Metrics}

The quality of tag clouds is usually assessed by the users that subjectively rate the structure and arrangement of tags in the cloud. However, such users based assessments are expensive and hardly available. To overcome this limitation, we use synthetic metrics for evaluation of different aspects of a generated tag cloud. Such metrics allow to measure the quality of tag clouds and, as a consequence, various tag selection algorithms can be utilized to maximize considered metrics. In this work, we consider 2 well-known metrics, coverage and overlap, introduced in 23. Furthermore, we introduce a new metric chained coverage which is utilized in the proposed methodologies. For the following definitions consider $D$ as a set of exiting documents, $T$ as the whole set of existing tags and $D_{t}$ as the set of documents assigned to a tag $t \in T$.

The first metric is coverage, defined as:

$$
\text { Coverage }(t)=\frac{\left|D_{t}\right|}{\left|D_{a}\right|},
$$

where $\left|D_{t}\right|$ is the number of documents assigned to a tag $t$ and $\left|D_{a}\right|$ is the number of all documents that are considered during a tag cloud generation process. The metric ranges between 0 and 1 . When a coverage for a particular tag $t$ is close to 1 , the majority of considered documents was annotated with a tag $t$. We utilize this metric during the selection process to maximize number of documents that can be accessed directly by exploring a tag cloud. 
Overlap of $T_{c}$ : Different tags in $T_{c}$ may be assigned with the same item in $D_{T_{c}}$. The overlap metric captures the extent of such redundancy. Thus, given $t_{i} \in T_{c}$ and $t_{j} \in T_{c}$, we define the overlap over $\left(T_{c}\right)$ of $T_{c}$ as:

$$
\operatorname{Overlap}\left(T_{c}\right)=\operatorname{avg}_{t_{i} \neq t_{j}} \frac{\left|D_{t_{i}} \cap D_{t_{j}}\right|}{\min \left\{\left|D_{t_{i}}\right|,\left|D_{t_{j}}\right|\right\}},
$$

If over $\left(T_{c}\right)$ is close to 0 , then the intersections of documents annotated by depicted tags are small and such tag clouds are more diverse.

There exist different selection techniques that try to optimize a given metrics which result into enriched tag clouds. In this work, we propose two new methodologies that improve introduced metrics. Furthermore, we introduce a new metric chained coverage that captures how many documents are covered by a considered tag given that documents covered by previously selected tags are not considered. This metric combines coverage and overlap altogether and provides simpler decision-making during the tag selection for the tag cloud. Chained coverage is given as:

$$
\text { Chained coverage }\left(t \mid T_{s}\right)=\frac{\left|D_{t} \backslash D_{T_{s}}\right|}{\left|D_{a}\right|},
$$

where $D_{T_{s}}$ is a set of documents covered by previously selected tags $T_{s}$. The proposed metric can be understood as combination of the classical coverage with the zero overlap with the respect to the previously selected tags. We assume that the diversity of the tag cloud is desired property as users are not interested in retrieving redundant documents covered by different tags. Therefore, the goal is to maximize a chained coverage of each tag used for the tag cloud generation. The metric simplifies a selection process of tags as instead of optimizing two independent metrics i.e., coverage and overlap we maximize only the chained coverage.

\subsection{Syntactical Pre-clustering of Tags}

Social tagging systems collect heterogeneous tags assigned by the users to the resources of the system. Tags in these systems can have the same semantical meaning however they are syntactically different i.e., typos, singular and plural forms and compounded tags. For example, when we look at the 20 most frequent tags of Delicious dataset (introduced in Section 5) and identified that they have at least 6 and at most 20 different syntactical alternatives in the whole tag space are present. Tags like Web_design, web-design, webDesign or *webdesign can be aggregated and represented only with the most frequent tag webdesign.

To remove from the tag cloud syntactically different tags with the same semantical meaning, we propose a methodology that aggregates syntactically similar tags into clusters. In the tag cloud generation process, obtained clusters can be represented only with the most frequent tag which can have the following benefits: 
- The coverage of the depicted tag in the tag cloud improve as it covers all documents annotated with the syntactically different tags from the given cluster

- Generated tag cloud does not contain syntactical variations of the same term as only the most frequent tag from each cluster is considered. Therefore, it allows to create a more diverse tag cloud, i.e., lower overlap between depicted tags.

In our method, syntactical pre-clustering introduced in Section 3.1 is used in the following manner. Levenhstein distance is first computed for each tag pair from the initial tag space. The edit distance between two tags measures the number of required changes (substitution, insertion and deletion of a character are allowed operations) to transform one tag into another. We justify its use because it attains significantly better results than Hamming distance as shown in [5].

Once, an edit distance is calculated, the tag space is divided into clusters. Each group contains only tags where the Levenhstein distance is equal or lower than a defined threshold (a number of maximum changes to transform a tag from the tag pair into a second tag). Then, the most frequent tag for each cluster is selected and is used in all further computations. It represents all other tags from a considered cluster.

In the end, our goal is that syntactical pre-clustering will affect the structure of the generated tag cloud in the sense that depicted tags are semantically more diverse.

\subsection{Improving Coverage and Diversity of Tag Clouds with Clustering}

The second methodology aggregates semantically related tags into a disjoint group. Each cluster can be perceived as a latent topic described with the related tags. The goal of cluster analysis is to cover all available topics in the tag space and as a consequence map it into a generated tag cloud to achieve maximal diversity of depicted tags. The methodology is motivated due to the drawback of the usual approach (denoted also as a baseline approach) where only the most frequent tags are considered. The selection of the most popular tags results into a tag cloud with terms that have too broad meaning. Therefore, depicted tags cover redundantly a certain set of documents i.e, the overlap of such tag cloud is unnecessary high. For instance a tag cloud generated from the top-25 most frequent tags from Bibsonomy dataset [13 contains tags as public, video, Media, books, blog or search. Obviously, such tags have general meaning or no information value for users. Moreover, often are assigned to the documents in combination with other frequent tags. The possible solution is to minimize a number of tags with the general meaning and additionally select popular but more specific tags as the objective is to preserve the coverage and minimize overlap of the tag cloud.

The aforementioned drawbacks of tag clouds generated from the most popular tags are addressed with the combination of cluster analysis of tags and 
maximization of the introduced metric - chained coverage. The former one provides basis for a diversity of a generated tag cloud by assuring that all latent topics within the tag space are captured. The latter one suppresses tags with the general meaning and instead selects popular but specific tags. The maximization of the chained coverage promotes (specific) tags with the high coverage of not yet covered documents by previously selected tags. On the other hand frequent tags with low chained coverage (general meaning) are omitted.

We explore different approaches of tags selection from the created clusters. The method based on selecting one tag with the highest coverage from each cluster generates more diverse tag clouds. However, the coverage is lower or comparable to the baseline approach as the chained coverage of generated clusters follows a power law distribution. Thus, majority of clusters belong to the long tail of such distribution.

Therefore, we propose a technique (see Algorithm 1) that selects tags proportionaly from each cluster. The provided tags are syntactically grouped and subsequently semantically clustered by one of the introduced clustering technique. The number of clusters is equal to the tag cloud size. The obtained clusters are sorted by chained coverage in descending order. The chained coverage of each cluster is given by previously explored clusters starting from cluster with the highest coverage. The method computes the number of tags to be selected from the cluster based on the chained coverage of a given cluster given the tag cloud size. From each cluster is selected a number of tags with the highest chained coverage. The goal is to cover a given cluster as good as possible in terms of coverage and overlap. The selection based on maximization of chained coverage satisfies such requirements. The method terminates when the number of selected tags is equal to the tag cloud size.

\subsection{Tag Cloud Generation}

Once, the tags are selected according to our proposed methodologies, they are depicted in the tag cloud. Semantically related tags from the same cluster are displayed with the same color which is specific for each cluster. Such tags are also located near each other and it allows to explore tags in more convenient way. Location and particular color of tags from the identical cluster results into a tag cloud which is semantically structured and as was shown in [8]. This presentation structure differs from the most common visualization of tag clouds where tags are alphabetically sorted. It allows to differentiate main topics in the tag cloud and also users can perceive and notice semantic relations between tags in neighbourhood [8]. Moreover, it helps to understand connections between tags, for example, tags cucumber and Spain are hardly interpretable in alphabetically sorted tag cloud. However, if they are depicted together with the tag E.coli a user can easily assume that these tags are related to E.coli outbreak.

Personalized Tag Cloud Generation: Another benefit of performed cluster analysis is a possibility to generate personalized tag clouds. Such tag cloud is an 
Input: tags, tagCloudSize

Output: selectedTags

$\mathbf{1}$ tags $\leftarrow$ syntacticalClustering(tags);

2 clusters $\leftarrow$ semanticalClustering(tags,tagCloudSize);

3 clusters $\leftarrow$ sortClustersByChainedCoverage(clusters);

4 foreach cluster in clusters do

$5 \quad$ tagsToSelect $\leftarrow$ cluster.chainedCoverage(exploredClusters) $\cdot$ tagCloudSize

6 for $i=1$ to tagsToSelect do

7 foreach tag in cluster do

8 if tag.chainedCoverage(selectedTags) is highest in the cluster then

$9 \quad \mid$ if tag.chainedCoverage(selectedTags) $>$ threshold then

10

11

12

13

14

15

16

17

18

end

selectedTags $\leftarrow$ selectedTags + tag;

end

end

if size of selectedTags $>$ tagCloudSize then

return [selectedTags]

end

exploredClusters $\leftarrow$ exploredClusters + cluster;

Algorithm 1. The methodology for tag cloud generation

adapted version of the above-mentioned general tag cloud model. User's preferences are incorporated into the tag cloud such that tags related to user's tags are preferred over others. The selection of similar tags is performed by retrieving tags from the clusters that contain at least one of the user's tags. However, in this work we do not evaluate proposed methodologies on the personalized tag clouds.

\section{Experiments}

We investigate the improvements of the proposed methodologies in terms of coverage and overlap of generated tag clouds. The proposed techniques are evaluated on the BibSonomy dataset [13 and the snapshot of Delicious dataset which consists of bookmarking activity on www.delicious.com from 8th till 16th of September 2009. Bibsonomy dataset contains 5794 distinct users, 802045 items and 204850 tags. The total number of tagging posts is 2555080 . The snapshot of Delicious dataset contains 187359 users, 185401 unique tags and 355525 bookmarks. The total number of tagging posts is 2046868 . The evaluation is conducted on the above-described datasets as they represent the most popular collaborative tagging systems of Web nowadays.

All the experiments are conducted on Ubuntu Server 11.10 64-bit operating system running on Intel Xeon X3460 CPU 2.8GHz with 8 GB RAM. The tag cloud generation methodologies and clustering techniques are implemented in Java 6 and source code and all results are available on our website1.

\footnotetext{
${ }^{1}$ http://people.cs.aau.dk/ mleginus/icwe2012/
} 


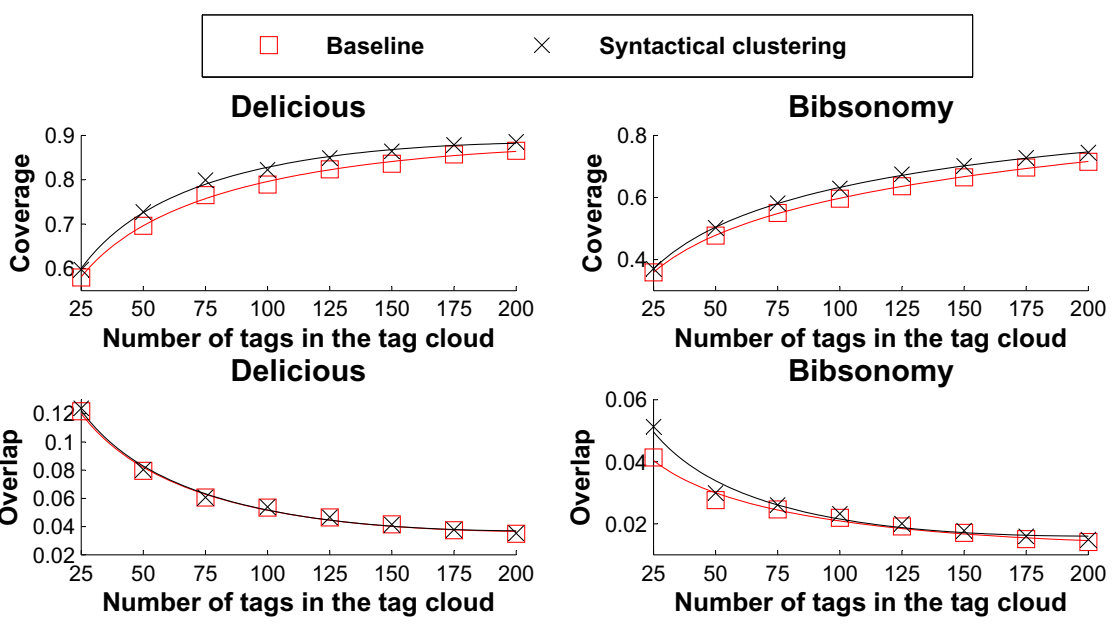

Fig. 1. Coverage and overlap results for baseline (red) and pre-clustering (black) methods and their corresponding logarithmic fit

\subsection{Syntactical Pre-clustering of Tags}

Table 1 summarizes the mean values of both methods in both datasets in terms of coverage and overlap. Results show that the coverage of the syntactical preclustering of tags is better than the baseline on the two datasets we tested. Coverage had a 5\% (5079 documents) increase on BiSonomy dataset and 3.5\% (3072 documents) increase on Delicious. Overlap, on the other hand, had similar results. Pre-clustering on BibSonomy had slightly higher mean but the values were practically the same on Delicious. One explanation but the higher means on

Table 1. Mean values of coverage and overlap for the baseline and syntactical preclustering methods on BibSonomy and Delicious datasets

\begin{tabular}{lcc|cc}
\hline \hline & \multicolumn{2}{c}{ Coverage } & \multicolumn{2}{c}{ Overlap } \\
\hline Dataset & Baseline & Pre-Clustering & Baseline & Pre-Clustering \\
BibSonomy & 0.586 & 0.616 & 0.022 & 0.025 \\
Delicious & 0.776 & 0.803 & 0.059 & 0.060 \\
\hline
\end{tabular}

Delicious is the different nature of both datasets. BibSonomy is a more specific dataset, containing domain-specific tags. Delicious, on the other hand, is used by a wider variety of users on different domains.

Next, we looked at how the two methodologies perform as a function of the number of tags in the tag cloud. Figure 1 shows these results. In both methods, as number of tags increases, coverage and overlap improves in a logarithmic fashion. In fact, the logarithmic curve fits almost perfectly (R-square $>0.98$, p-value $<0.001)$ in all cases. That means that coverage and overlap improve significantly after tag clouds with 75 tags but then stabilizes. Note that coverage of the syntactical pre-clustering improves over the baseline as the number of 
tags increase. This is specially the case in the BibSonomy dataset. In the case of overlap, the values stay almost identical on the Delicious dataset. On BibSonomy, overlap is worse with the pre-clustering method for tag clouds with few tags but improves as the number of tags increase.

Overall, these results indicate that by using the pre-clustering method, coverage of tag clouds improve. We consider it positive to note that overlap remains similar to the baseline as the number of tags in the tag cloud increases. This indicates a solid improvement of the coverage of the tag clouds generated.

\subsection{Improving Coverage and Diversity of Tag Clouds with Clustering}

We compare the proposed methodology with the baseline algorithm on both datasets. In this work, the tag cloud generation considers the whole tag space and all available resources of the social tagging systems. However, the generated tag cloud consists from at most 200 tags and not frequent tags can be omitted. Therefore, without loss of generality, we prune considered datasets. The evaluated methods utilize tags that were assigned by users at least 50 times and related documents that were annotated at least 5 times. For both techniques, we iteratively increase a number of tags in the tag cloud starting with 25 till 200 tags with the step 25. The results are presented in the following Figure. 2 The proposed methodology improves the coverage on both datasets. Similarly, the overlap of generated tag clouds is decreased. The best performing clustering technique is hierarchical clustering which computes a tag pairs co-occurrences. The obtained clusters consist of semantically related tags and as consequence

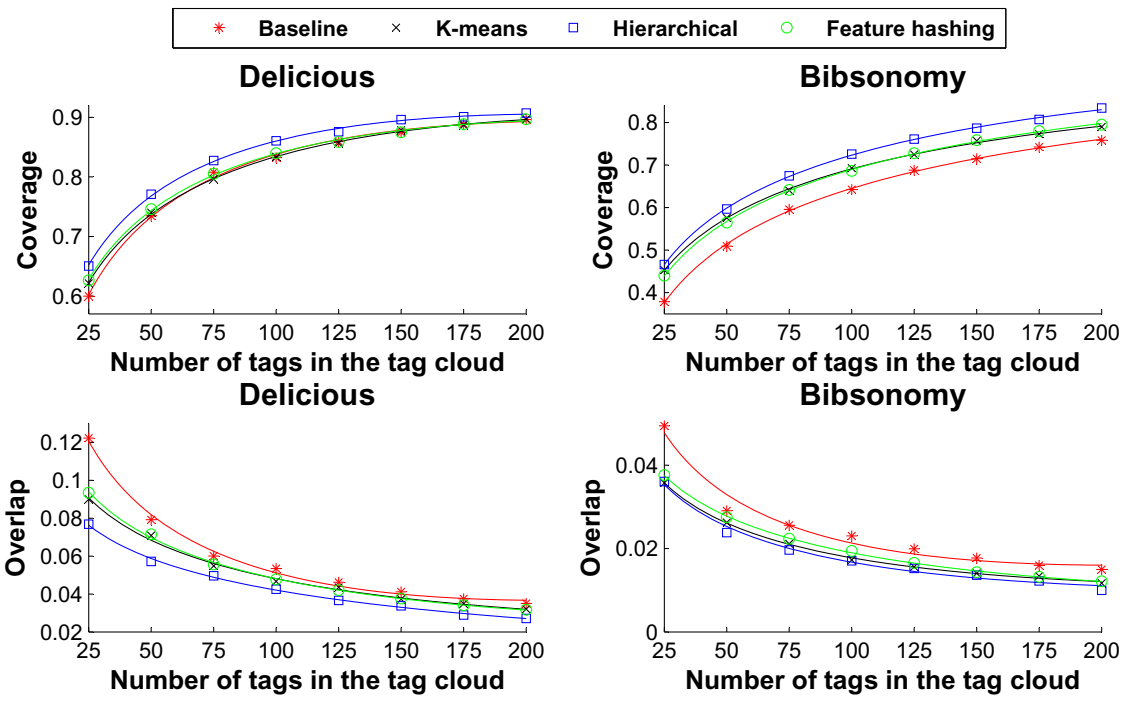

Fig. 2. Improvements of coverage and overlap on Bibsonomy and Delicious datasets with different clustering techniques and their corresponding logarithmic fit 
a tag space is uniformly covered by these clusters. Feature hashing improves metrics insignificantly in comparison to the baseline method. The drawback of this clustering method is that aggregates all tags to the top- $k$ most frequent tags which can be considered as centroids of generated clusters. The most frequent tags are often semantically similar and it negatively affects the structure of obtained clusters. K-means clustering represents each tag as a feature vector. and the size of features is number of documents. As the number of features is enormous, the computation of distances between tags results into a creation of clusters with semantically not related tags. Another drawback is the random placement of the initial centroids of clusters that significantly affects a final structure of clusters.

In average, the proposed methodology improves the coverage with $12.4 \%$ on Delicious and $3.01 \%$ on Bibsonomy dataset. The improvements of coverage can be expressed also with the number of additionally covered documents. The tag clouds generated by the methodology cover additional 2794 documents on Delicious and 56508 documents on Bibsonomy. The diversity of tag clouds is also improved which can be observed by decreased overlap

The average improvements are presented in the following Tables 2 and 3. The main reason of improvements is the introduced maximization of chained

Table 2. Mean values of coverage for the baseline and different clustering methods on BibSonomy and Delicious datasets

\begin{tabular}{l|cccc}
\hline \hline & \multicolumn{4}{|c}{ Coverage } \\
\hline Dataset & Baseline & K-means & Hierarchical & Feature hashing \\
BibSonomy & 0.6285 & 0.6756 & 0.7064 & 0.6741 \\
Delicious & 0.8112 & 0.8139 & 0.8362 & 0.8171 \\
\hline
\end{tabular}

Table 3. Mean values of overlap for the baseline and different clustering methods on BibSonomy and Delicious datasets

\begin{tabular}{|c|c|c|c|c|}
\hline & \multicolumn{4}{|c|}{ Overlap } \\
\hline Dataset & Baseline & K-means & Hierarchi & Feature hashing \\
\hline BibSonomy & 0.0245 & 0.0194 & 0.0185 & 0.0204 \\
\hline Delicious & 0.0731 & 0.0543 & 0.0637 & 0.0631 \\
\hline
\end{tabular}

coverage. The metric significantly simplifies the selection process of tags for the tag cloud as it maximizes coverage and minimizes overlap of generated tag cloud altogether. The overall coverage is equal to the chained coverage of the whole tag cloud. Therefore, the presentation of the chained coverage is unnecessary however, it is a core factor of achieved improvements.

Although the experiments were carried out using the Delicious and Bibsonomy dataset, other datasets should be also evaluated. Statistical properties of each dataset differ therefore, we assume that our methods could be succesfully aplied on other folksonomy based datasets. However, this needs to be empirically proved. 


\section{Conclusion and Future Work}

In this work, we propose two different methodologies for an enhanced tag cloud generation. The former one improves the coverage of the tag clouds by aggregation of syntactically similar tags. Moreover, it prohibits a depiction of the syntactically similar tags and as a consequence additional tags can be selected for the generated tag cloud. It results into more diverse structure of the tag clouds. The latter methodology improves coverage and decreases overlap of tag clouds. Introduced metric - chained coverage simplifies a selection process and the optimization of coverage and overlap is straightforward. The utilization of clustering techniques allow to divide the tag space into disjoint groups which allows to select more diverse tags from the obtained clusters. The best improvements are attained with hierarchical clustering that computes tag pairs co-occurrences and produces the most reasonable clusters. The proposed methodology produces tag clouds that cover As a future work we intend to explore possible new metrics that would incorporate well-known metrics altogether and in a such way simplify a selection process of tags.

Acknowledgements. This work has been supported by FP7 ICT project M-Eco: Medical Ecosystem Personalized Event-Based Surveillance under grant No. 247829.

\section{References}

1. Bai, B., Weston, J., Grangier, D., Collobert, R., Sadamasa, K., Qi, Y., Chapelle, O., Weinberger, K.: Learning to rank with (a lot of) word features. Information Retrieval 13(3), 291-314 (2010)

2. Bateman, S., Gutwin, C., Nacenta, M.: Seeing things in the clouds: the effect of visual features on tag cloud selections. In: Proceedings of the Nineteenth ACM Conference on Hypertext and Hypermedia, HT 2008, pp. 193-202. ACM, New York (2008)

3. Bateman, S., Gutwin, C., Nacenta, M.: Seeing things in the clouds: the effect of visual features on tag cloud selections. In: Proceedings of the Nineteenth ACM Conference on Hypertext and Hypermedia, HT 2008, pp. 193-202. ACM, New York (2008)

4. Durao, F., Dolog, P., Leginus, M., Lage, R.: SimSpectrum: A Similarity Based Spectral Clustering Approach to Generate a Tag Cloud. In: Harth, A., Koch, N. (eds.) ICWE 2011. LNCS, vol. 7059, pp. 145-154. Springer, Heidelberg (2012)

5. Echarte, F., Astrain, J.J., Córdoba, A., Villadangos, J.: Pattern Matching Techniques to Identify Syntactic Variations of Tags in Folksonomies. In: Lytras, M.D., Damiani, E., Tennyson, R.D. (eds.) WSKS 2008. LNCS (LNAI), vol. 5288, pp. 557-564. Springer, Heidelberg (2008)

6. Halvey, M.J., Keane, M.T.: An assessment of tag presentation techniques. In: Proceedings of the 16th International Conference on World Wide Web, WWW 2007, pp. 1313-1314. ACM, New York (2007)

7. Hassan-Montero, Y., Herrero-Solana, V.: Improving tag-clouds as visual information retrieval interfaces. In: INSCIT 2006 Conference, Merída (2006) 
8. Hassan-Montero, Y., Herrero-Solana, V.: Improving tag-clouds as visual information retrieval interfaces. In: International Conference on Multidisciplinary Information Sciences and Technologies, Citeseer, pp. 25-28 (2006)

9. Huang, A.: Similarity measures for text document clustering. In: Proceedings of the Sixth New Zealand Computer Science Research Student Conference (NZCSRSC 2008), Christchurch, New Zealand, pp. 49-56 (2008)

10. Johnson, S.: Hierarchical clustering schemes. Psychometrika 32(3), 241-254 (1967)

11. Kaser, O., Lemire, D.: Tag-cloud drawing: Algorithms for cloud visualization. CoRR, abs/cs/0703109 (2007)

12. Knautz, K., Soubusta, S., Stock, W.G.: Tag clusters as information retrieval interfaces. In: HICSS, pp. 1-10 (2010)

13. Knowledge and U. o. K. Data Engineering Group: Benchmark folksonomy data from bibsonomy, version of January 1 (2010)

14. Kuo, B.Y.-L., Hentrich, T., Good, B.M., Wilkinson, M.D.: Tag clouds for summarizing web search results. In: Proceedings of the 16th International Conference on World Wide Web, WWW 2007, pp. 1203-1204. ACM, New York (2007)

15. Kuo, B.Y.-L., Hentrich, T., Good, B.M., Wilkinson, M.D.: Tag clouds for summarizing web search results. In: Proceedings of the 16th International Conference on World Wide Web, WWW 2007, pp. 1203-1204. ACM, New York (2007)

16. Leginus, M., Zemaitis, V.: Speeding up tensor based recommenders with clustered tag space and improving quality of recommendations with non-negative tensor factorization. Master's thesis, Aalborg University (2011)

17. Ramage, D., Heymann, P., Manning, C., Garcia-Molina, H.: Clustering the tagged web. In: Proceedings of the Second ACM International Conference on Web Search and Data Mining, pp. 54-63. ACM (2009)

18. Rivadeneira, A.W., Gruen, D.M., Muller, M.J., Millen, D.R.: Getting our head in the clouds: toward evaluation studies of tagclouds. In: Proceedings of the SIGCHI Conference on Human factors in Computing Systems, CHI 2007, pp. 995-998. ACM, New York (2007)

19. Schrammel, J., Leitner, M., Tscheligi, M.: Semantically structured tag clouds: an empirical evaluation of clustered presentation approaches. In: Proceedings of the 27th International Conference on Human Factors in Computing Systems, CHI 2009, pp. 2037-2040. ACM, New York (2009)

20. Shepitsen, A., Gemmell, J., Mobasher, B., Burke, R.: Personalized recommendation in social tagging systems using hierarchical clustering. In: Proceedings of the 2008 ACM Conference on Recommender Systems, RecSys 2008, pp. 259-266. ACM, New York (2008)

21. Sinclair, J., Cardew-Hall, M.: The folksonomy tag cloud: when is it useful? J. Inf. Sci. 34, 15-29 (2008)

22. van Dam, J., Vandic, D., Hogenboom, F., Frasincar, F.: Searching and browsing tag spaces using the semantic tag clustering search framework. In: 2010 IEEE Fourth International Conference on Semantic Computing (ICSC), pp. 436-439. IEEE (2010)

23. Venetis, P., Koutrika, G., Garcia-Molina, H.: On the selection of tags for tag clouds. In: Proceedings of the Fourth ACM International Conference on Web Search and Data Mining, WSDM 2011, pp. 835-844 (2011) 